\title{
James' Submodule Theorem and the Steinberg Module
}

\section{Meinolf GECK}

IAZ - Lehrstuhl für Algebra, Universität Stuttgart, Pfaffenwaldring 57, D-70569 Stuttgart, Germany

E-mail: meinolf.geck@mathematik.uni-stuttgart.de

URL: http://www. mathematik. uni-stuttgart.de/ geckmf /

Received August 29, 2017, in final form November 28, 2017; Published online December 05, 2017 https://doi.org/10.3842/SIGMA.2017.091

\begin{abstract}
James' submodule theorem is a fundamental result in the representation theory of the symmetric groups and the finite general linear groups. In this note we consider a version of that theorem for a general finite group with a split $B N$-pair. This gives rise to a distinguished composition factor of the Steinberg module, first described by Hiss via a somewhat different method. It is a major open problem to determine the dimension of this composition factor.
\end{abstract}

Key words: groups with a $B N$-pair; Steinberg representation; modular representations

2010 Mathematics Subject Classification: 20C33; 20C20

\section{Introduction}

Let $G$ be a finite group of Lie type and $\mathrm{St}_{k}$ be the Steinberg representation of $G$, defined over an arbitrary field $k$; see [8]. We shall be concerned here with the case where $\mathrm{St}_{k}$ is reducible. There is only very little general knowledge about the structure of $\mathrm{St}_{k}$ in this case; see, e.g., [3, 4] and the references there. Using the theory of Gelfand-Graev representations of $G$, Hiss [5] showed that $\mathrm{St}_{k}$ always has a certain distinguished composition factor with multiplicity 1. It appears to be extremely difficult to determine further properties of this composition factor, e.g., its dimension. The purpose of this note is to show that this composition factor can be defined in a somewhat more intrinsic way through a version of James' submodule theorem [6]; see Remark 3.4.

\section{Groups with a split $B N$-pair}

Let $G$ be a finite group and $B, N \subseteq G$ be subgroups which satisfy the axioms for an "algebraic group with a split $B N$-pair" in [1, Section 2.5]. We just recall explicitly those properties of $G$ which will be important for us in the sequel. Firstly, there is a prime number $p$ such that we have a semidirect product decomposition $B=U \rtimes H$ where $H=B \cap N$ is an abelian group of order prime to $p$ and $U$ is a normal $p$-subgroup of $B$. The group $H$ is normal in $N$ and $W=N / H$ is a finite Coxeter group with a canonically defined generating set $S$; let $l: W \rightarrow \mathbb{N}_{0}$ be the corresponding length function. For each $w \in W$, let $n_{w} \in N$ be such that $H n_{w}=w$. Let $w_{0} \in W$ be the unique element of maximal length; we have $w_{0}^{2}=1$. Let $n_{0} \in N$ be a representative of $w_{0}$ and $V:=n_{0}^{-1} U n_{0}$; then $U \cap V=H$. For $w \in W$, let $U_{w}:=U \cap n_{w}^{-1} V n_{w}$.

This paper is a contribution to the Special Issue on the Representation Theory of the Symmetric Groups and Related Topics. The full collection is available at https://www.emis.de/journals/SIGMA/symmetric-groups2018.html 
(Note that $V, U_{w}$ do not depend on the choices of $n_{0}, n_{w}$.) Then we have the following sharp form of the Bruhat decomposition:

$$
G=\coprod_{w \in W} B n_{w} U_{w}, \quad \text { with uniqueness of expressions, }
$$

that is, every $g \in B n_{w} B$ can be uniquely written as $g=b n_{w} u$ where $b \in B$ and $u \in U_{w}$. Note that, since $w_{0}^{2}=1$, we have $U_{w_{0}}=U$ and $B n_{0} B=B n_{0} U=U n_{0} B$, in both cases with uniqueness of expressions.

Let $k$ be any field and $k G$ be the group algebra of $G$. All our $k G$-modules will be finitedimensional and left modules.

Remark 2.1. Let $\underline{\mathfrak{b}}:=\sum_{b \in B} b \in k G$. Then $k G \underline{\mathfrak{b}}$ is a left $k G$-module which is canonically isomorphic to the induced module $\operatorname{Ind}_{B}^{G}\left(k_{B}\right)$; here, $k_{B}$ denotes the trivial $k B$-module. Now $k G \underline{\mathfrak{b}}$ carries a natural symmetric bilinear form $\langle\rangle:, k G \underline{\mathfrak{b}} \times k G \underline{\mathfrak{b}} \rightarrow k$ such that, for any $g, g^{\prime} \in G$, we have

$$
\left\langle g \underline{\mathfrak{b}}, g^{\prime} \underline{\mathfrak{b}}\right\rangle= \begin{cases}1, & \text { if } g B=g^{\prime} B \\ 0, & \text { otherwise }\end{cases}
$$

This form is non-singular and $G$-invariant. For any subset $X \subseteq k G \underline{\mathfrak{b}}$, we denote

$$
X^{\perp}:=\{a \in k G \underline{\mathfrak{b}} \mid\langle a, x\rangle=0 \text { for all } x \in X\} .
$$

If $X$ is a $k G$-submodule of $k G \underline{\mathfrak{b}}$, then so is $X^{\perp}$.

Remark 2.2. Let $\sigma: U \rightarrow k^{\times}$be a group homomorphism and set

$$
\underline{\mathfrak{u}}_{\sigma}=\sum_{u \in U} \sigma(u) u \in k G
$$

Then $\Gamma_{\sigma}:=k G \underline{\mathfrak{u}}_{\sigma}$ is a left $k G$-module which is isomorphic to the induced module $\operatorname{Ind}_{U}^{G}\left(k_{\sigma}\right)$, where $k_{\sigma}$ denotes the 1-dimensional $k U$-module corresponding to $\sigma$. Note that $\underline{\mathfrak{u}}_{\sigma}^{2}=|U| \underline{\mathfrak{u}}_{\sigma}$. Hence, if $|U| 1_{k} \neq 0$, then $\Gamma_{\sigma}$ is a projective $k G$-module.

We say that $\sigma$ is non-degenerate if the restriction of $\sigma$ to $U_{s}$ is non-trivial for every $s \in S$. Note that this can only occur if $|U| 1_{k} \neq 0$. In the case where $G=\mathrm{GL}_{n}(q)$, the following result is contained in James [6, Theorem 11.7(ii)]; see also Szechtman [9, Note 4.9, Section 10].

Lemma 2.3. Assume that $|U| 1_{k} \neq 0$ and $\sigma$ is non-degenerate. Then the subspace $\underline{\mathfrak{u}}_{\sigma} k G \underline{\mathfrak{b}}$ is 1-dimensional and spanned by $\underline{\underline{u}}_{\sigma} n_{0} \underline{\mathfrak{b}}$. Furthermore, $\underline{\mathfrak{u}}_{\sigma} n_{w} \underline{\mathfrak{b}}=0$ for all $w \in W$ such that $w \neq w_{0}$.

Proof. By the Bruhat decomposition, we can write any $g \in G$ in the form $g=u n_{w} b$ where $u \in U, w \in W$ and $b \in B$. Now note that $b \underline{\mathfrak{b}}=\underline{\mathfrak{b}}$ for all $b \in B$ and $\underline{\mathfrak{u}}_{\sigma} u=\sigma(u)^{-1} \underline{\mathfrak{u}}_{\sigma}$ for all $u \in U$. Thus, $\underline{\mathfrak{u}}_{\sigma} k G \underline{\mathfrak{b}}$ is spanned by $\left\{\underline{\mathfrak{u}}_{\sigma} n_{w} \underline{\mathfrak{b}} \mid w \in W\right\}$. Now let $w \in W$ be such that $w \neq w_{0}$. We shall show that $\underline{\mathfrak{u}}_{\sigma} n_{w} \underline{\mathfrak{k}}=0$. For this purpose, we use the factorisation $U=U_{w} U_{w_{0} w}$ where $U_{w} \cap U_{w_{0} w}=\{1\}$; see [1, Proposition 2.5.12]. Since $w \neq w_{0}$, there exists some $s \in S$ such that $l(w s)>l(w)$ and so $U_{s} \subseteq U_{w_{0} w}$; see [1, Propositions 2.5.7(i) and 2.5.10(i)]. (Note that $U_{s}$ is denoted by $X_{i}$ in [loc. cit.].) Since $n_{w^{-1}} \in n_{w}^{-1} H$, we obtain

$$
\underline{\mathfrak{u}}_{\sigma} n_{w^{-1}} \underline{\mathfrak{b}}=\underline{\mathfrak{u}}_{\sigma} n_{w}^{-1} \underline{\mathfrak{b}}=\left(\sum_{u_{1} \in U_{w}} \sigma\left(u_{1}\right) u_{1}\right) n_{w}^{-1}\left(\sum_{u_{2} \in U_{w_{0}} w} \sigma\left(u_{2}\right) n_{w} u_{2} n_{w}^{-1} \underline{\mathfrak{b}}\right) .
$$


By the definition of $U_{w_{0} w}$, we have $n_{w} U_{w_{0} w} n_{w}^{-1} \subseteq U$ and so $n_{w} u_{2} n_{w}^{-1} \underline{\mathfrak{b}}=\underline{\mathfrak{b}}$ for every fixed $u_{2} \in U_{w_{0} w}$. Hence, we obtain

$$
\sum_{u_{2} \in U_{w_{0} w}} \sigma\left(u_{2}\right) n_{w} u_{2} n_{w}^{-1} \underline{\mathfrak{b}}=\left(\sum_{u_{2} \in U_{w_{0} w}} \sigma\left(u_{2}\right)\right) \underline{\mathfrak{b}} .
$$

Finally, since $U_{s} \subseteq U_{w_{0} w}$ and the restriction of $\sigma$ to $U_{s}$ is non-trivial, the above sum evaluates to 0 . Thus, $\underline{\mathfrak{u}}_{\sigma} n_{w^{-1}} \underline{\mathfrak{b}}=0$ for all $w \neq w_{0}$. Since $w_{0}=w_{0}^{-1}$, this also implies that $\underline{\mathfrak{u}}_{\sigma} n_{w} \underline{\mathfrak{b}}=0$ for all $w \neq w_{0}$, as required.

Hence, $\underline{\mathfrak{u}}_{\sigma} k G \underline{\mathfrak{b}}$ is spanned by $\underline{\mathfrak{u}}_{\sigma} n_{0} \underline{\mathfrak{b}}$. Finally, by the sharp form of the Bruhat decomposition, every element of $B n_{0} B$ has a unique expression of the form $u n_{0} b$ where $u \in U$ and $b \in B$. In particular, $\underline{\mathfrak{u}}_{\sigma} n_{0} \underline{\mathfrak{b}} \neq 0$ and so $\operatorname{dim} \underline{\mathfrak{u}}_{\sigma} k G \underline{\mathfrak{b}}=1$.

Corollary 2.4. Assume that $|U| 1_{k} \neq 0$ and $\sigma$ is non-degenerate. Then the map $\varphi: \Gamma_{\sigma} \rightarrow k G \underline{\mathfrak{b}}$, $\gamma \mapsto \gamma n_{0} \underline{\mathfrak{b}}$, is a non-zero $k G$-module homomorphism and every homomorphism $\Gamma_{\sigma} \rightarrow k G \underline{\mathfrak{b}}$ is a scalar multiple of $\varphi$.

Proof. The fact that $\varphi$, as defined above, is a $k G$-module homomorphism is clear; it is nonzero since $\varphi\left(\underline{\mathfrak{u}}_{\sigma}\right)=\underline{\mathfrak{u}}_{\sigma} n_{0} \underline{\mathfrak{b}} \neq 0$ by Lemma 2.3. Since $\underline{\mathfrak{u}}_{\sigma}$ is a non-zero scalar multiple of an idempotent (see Remark 2.2), we have $\operatorname{Hom}_{k G}\left(\Gamma_{\sigma}, k G \underline{\mathfrak{b}}\right) \cong \underline{\mathfrak{u}}_{\sigma} k G \underline{\mathfrak{b}}$ and this is 1-dimensional by Lemma 2.3.

\section{The submodule theorem}

We keep the notation of the previous section and assume throughout that $|U| 1_{k} \neq 0$. For any group homomorphism $\sigma: U \rightarrow k^{\times}$, we denote by $\sigma^{*}: U \rightarrow k^{\times}$the group homomorphism given by $\sigma^{*}(u)=\sigma(u)^{-1}$ for all $u \in U$. Note that, if $\sigma$ is non-degenerate, then so is $\sigma^{*}$. We can now state the following version of James's "submodule theorem" [6, Theorem 11.2], [6, 11.12(ii)].

Proposition 3.1 (cf. James [6, Theorem 11.2]). Let $\sigma: U \rightarrow k^{\times}$be non-degenerate and consider the submodule $\mathscr{S}_{\sigma}:=k G \underline{\mathfrak{u}}_{\sigma} n_{0} \underline{\mathfrak{b}} \subseteq k G \underline{\mathfrak{b}}$. Then the following hold.

(i) If $M \subseteq k G \underline{\mathfrak{b}}$ is any submodule, then either $\mathscr{S}_{\sigma} \subseteq M$ or $M \subseteq \mathscr{S}_{\sigma^{*}}^{\perp}$.

(ii) We have $\mathscr{S}_{\sigma} \nsubseteq \mathscr{S}_{\sigma^{*}}^{\perp}$ and $\mathscr{S}_{\sigma} \cap \mathscr{S}_{\sigma^{*}}^{\perp} \varsubsetneqq \mathscr{S}_{\sigma}$ is the unique maximal submodule of $\mathscr{S}_{\sigma}$.

(iii) The $k G$-module $D_{\sigma}:=\mathscr{S}_{\sigma} /\left(\mathscr{S}_{\sigma} \cap \mathscr{S}_{\sigma^{*}}^{\perp}\right)$ is absolutely irreducible and isomorphic to the contragredient dual of $D_{\sigma^{*}}$.

(iv) $D_{\sigma}$ occurs with multiplicity 1 as a composition factor of $k G \underline{\mathfrak{b}}$ and of $\mathscr{S}_{\sigma}$.

Proof. Having established Lemma 2.3 and Corollary 2.4, this readily follows from the general results in [6, Chapter 11]; the only difference is that James also assumes that $\mathscr{S}_{\sigma}=\mathscr{S}_{\sigma^{*}}$. As our notation and setting are somewhat different from those in [6], we recall the most important steps of the argument.

(i) Since $M \subseteq k G \underline{\mathfrak{b}}$, it follows from Lemma 2.3 that, for any $m \in M$, there exists some $c_{m} \in k$ such that $\underline{\mathfrak{u}}_{\sigma} m=c_{m} \underline{\underline{u}}_{\sigma} n_{0} \underline{\mathfrak{b}}$. If there exists some $m \in M$ with $c_{m} \neq 0$, then $\underline{\mathfrak{u}}_{\sigma} n_{0} \underline{\mathfrak{b}}=c_{m}^{-1} \underline{\mathfrak{u}}_{\sigma} m \in M$ and, hence, we have $\mathscr{S}_{\sigma} \subseteq M$ in this case. Now assume that $c_{m}=0$ for all $m \in M$; that is, we have $\underline{\mathfrak{u}}_{\sigma} M=\{0\}$. Let $m \in M$ and $g \in G$. Using the definition of $\underline{\mathfrak{u}}_{\sigma}, \underline{\mathfrak{u}}_{\sigma^{*}}$ and the $G$-invariance of $\langle$,$\rangle , we obtain$

$$
\left\langle m, g \underline{\mathfrak{u}}_{\sigma^{*}} n_{0} \underline{\mathfrak{b}}\right\rangle=\left\langle g^{-1} m, \underline{\mathfrak{u}}_{\sigma^{*}} n_{0} \underline{\mathfrak{b}}\right\rangle=\left\langle\underline{\mathfrak{u}}_{\sigma}\left(g^{-1} m\right), n_{0} \underline{\mathfrak{b}}\right\rangle=0,
$$

where the last equality holds since $\underline{\mathfrak{u}}_{\sigma} M=\{0\}$. Thus, $M \subseteq \mathscr{S}_{\sigma^{*}}^{\perp}$ in this case. 
(ii) For $u, u^{\prime} \in U$, we have $u n_{0} B=u^{\prime} n_{0} B$ if and only if $u=u^{\prime}$, by the sharp form of the Bruhat decomposition. Thus, we obtain

$$
\left\langle\underline{\mathfrak{u}}_{\sigma} n_{0} \underline{\mathfrak{k}}, \underline{\mathfrak{u}}_{\sigma^{*}} n_{0} \underline{\mathfrak{b}}\right\rangle=\sum_{u, u^{\prime} \in U} \sigma(u) \sigma^{*}\left(u^{\prime}\right)\left\langle u n_{0} \underline{\mathfrak{b}}, u^{\prime} n_{0} \underline{\mathfrak{b}}\right\rangle=|U| 1_{k} \neq 0,
$$

which means that $\mathscr{S}_{\sigma} \nsubseteq \mathscr{S}_{\sigma^{*}}^{\perp}$ and so $\mathscr{S}_{\sigma} \cap \mathscr{S}_{\sigma^{*}}^{\perp} \varsubsetneqq \mathscr{S}_{\sigma}$. Now let $M \varsubsetneqq \mathscr{S}_{\sigma}$ be any maximal submodule. Then (i) immediately implies that $M=\mathscr{S}_{\sigma} \cap \mathscr{S}_{\sigma^{*}}^{\perp}$.

(iii) By (ii), we already know that $D_{\sigma}$ is irreducible. The remaining assertions then follow exactly as in the proof of [6, Theorem 11.2].

(iv) By construction, $D_{\sigma}$ occurs at least once in $k G \underline{\mathfrak{b}}$ and in $\mathscr{S}_{\sigma}$. Let $\varphi: \Gamma_{\sigma} \rightarrow k G \underline{\mathfrak{b}}$ be as in Corollary 2.4. Since $\Gamma_{\sigma}$ is projective and $\varphi\left(\Gamma_{\sigma}\right)=\mathscr{S}_{\sigma}$, some indecomposable direct summand of $\Gamma_{\sigma}$ is a projective cover of $D_{\sigma}$ and so the desired multiplicity of $D_{\sigma}$ is at most $\operatorname{dim} \operatorname{Hom}_{k G}\left(\Gamma_{\sigma}, k G \underline{\mathfrak{k}}\right)=1$, where the last equality holds by Corollary 2.4 .

We now relate the modules $\mathscr{S}_{\sigma}, D_{\sigma}$ in Proposition 3.1 to the Steinberg module $\mathrm{St}_{k}$ of $G$, as defined in [8]. Recall that

$$
\mathrm{St}_{k}=k G \mathfrak{e} \subseteq k G \underline{\mathfrak{k}}, \quad \text { where } \quad \mathfrak{e}:=\sum_{w \in W}(-1)^{l(w)} n_{w} \underline{\mathfrak{b}} .
$$

We have $\operatorname{dim} \mathrm{St}_{k}=|U|$; a basis of $\operatorname{St}_{k}$ is given by $\{u \mathfrak{e} \mid u \in U\}$.

Proposition 3.2. We have $\mathscr{S}_{\sigma}=k G \underline{\mathfrak{u}}_{\sigma} \mathfrak{e} \subseteq \mathrm{St}_{k}$. Consequently, $D_{\sigma}$ is a composition factor (with multiplicity 1) of $\mathrm{St}_{k}$.

Proof. By Lemma 2.3, we have the identity

$$
\underline{\mathfrak{u}}_{\sigma} \mathfrak{e}=\sum_{w \in W}(-1)^{l(w)} \underline{\mathfrak{u}}_{\sigma} n_{w} \underline{\mathfrak{h}}=(-1)^{l\left(w_{0}\right)} \underline{\mathfrak{u}}_{\sigma} n_{0} \underline{\mathfrak{k}}
$$

and so $\mathscr{S}_{\sigma}=k G \underline{\mathfrak{u}}_{\sigma} n_{0} \underline{\mathfrak{b}}=k G \underline{\mathfrak{u}}_{\sigma} \mathfrak{e} \subseteq k G \mathfrak{e}=\mathrm{St}_{k}$. The statement about $D_{\sigma}$ then follows from Proposition 3.1(iv).

Remark 3.3. Gow [4, Section 2] gives an explicit formula for the restriction of the bilinear form $\langle\rangle:, k G \underline{\mathfrak{b}} \times k G \underline{\mathfrak{b}} \rightarrow k$ (see Remark 2.1) to $\mathrm{St}_{k}$. For this purpose, he first works over $\mathbb{Z}$ and then rescales to obtain a non-zero form over $k$. One can also proceed directly, as follows. We have

$$
\left\langle u_{1} \mathfrak{e}, u_{2} \mathfrak{e}\right\rangle=c_{W}\left(u_{1}^{-1} u_{2}\right) 1_{k} \quad \text { for any } u_{1}, u_{2} \in U
$$

where $c_{W}(u):=\left|\left\{w \in W \mid n_{w}^{-1} u n_{w} \in U\right\}\right|$ for $u \in U$. Indeed, since $\langle$,$\rangle is G$-invariant, it is enough to show that $\langle\mathfrak{e}, u \mathfrak{e}\rangle=c_{W}(u) 1_{k}$ for $u \in U$. Now, we have

$$
\langle\mathfrak{e}, u \mathfrak{e}\rangle=\sum_{w, w^{\prime} \in W}(-1)^{l(w)+l\left(w^{\prime}\right)}\left\langle n_{w} \underline{\mathfrak{k}}, u n_{w^{\prime}} \underline{\mathfrak{k}}\right\rangle=\sum_{w \in W}\left\langle n_{w} \underline{\mathfrak{k}}, u n_{w} \underline{\mathfrak{b}}\right\rangle,
$$

where the second equality holds since $n_{w} B=u n_{w^{\prime}} B$ if and only if $w=w^{\prime}$. Furthermore, $n_{w} B=u n_{w} B$ if and only if $n_{w}^{-1} u n_{w} \in B$. Since $n_{w}^{-1} u n_{w}$ is a $p$-element, the latter condition is equivalent to $n_{w}^{-1} u n_{w} \in U$. This yields the desired formula.

Remark 3.4. Since $|U| 1_{k} \neq 0$, the module $\Gamma_{\sigma}$ in Remark 2.2 is projective. Also note that $\mathscr{S}_{\sigma}=\varphi\left(\Gamma_{\sigma}\right)$ where $\varphi$ is defined in Corollary 2.4. Using also Proposition 3.2, we conclude that $\operatorname{dim} \operatorname{Hom}_{k G}\left(\Gamma_{\sigma}, \mathrm{St}_{k}\right)=\operatorname{dim} \operatorname{Hom}_{k G}\left(\Gamma_{\sigma}, k G \underline{\mathfrak{k}}\right)=1$. So there is a unique indecomposable direct summand $P_{\mathrm{St}}$ of $\Gamma_{\sigma}$ such that

$$
\operatorname{Hom}_{k G}\left(P_{\mathrm{St}}, \mathrm{St}_{k}\right) \neq 0
$$


Being projective indecomposable, $P_{\mathrm{St}}$ has a unique simple quotient whose Brauer character is denoted by $\sigma_{G}$ by Hiss [5, Section 6]. By Proposition 3.1(iv) (and its proof), we now see that $\sigma_{G}$ is the Brauer character of $D_{\sigma}$.

Remark 3.5. It is known that the socle of $\mathrm{St}_{k}$ is simple; see $[3,10]$. We claim that this simple socle is contained in $\mathscr{S}_{\sigma} \cap \mathscr{S}_{\sigma^{*}}^{\perp}$, unless $\mathrm{St}_{k}$ is irreducible. Indeed, assume that $\mathscr{S}_{\sigma} \cap \mathscr{S}_{\sigma^{*}}^{\frac{1}{*}}=\{0\}$. Then $D_{\sigma} \subseteq \mathrm{St}_{k} \subseteq k G \underline{\mathfrak{b}}$ and, hence, $D_{\sigma}$ belongs to the Harish-Chandra series defined by the pair $\left(\varnothing, k_{H}\right)$ (notation of Hiss [5, Theorem 5.8]). By Remark 3.4 and the argument in [5, Lemma 6.2], it then follows that $[G: B] 1_{k} \neq 0$. So $\mathrm{St}_{k}$ is irreducible by [8].

\section{Examples}

We keep the setting of the previous section. We also assume now that $G$ is a true finite group of Lie type, as in [1, Section 1.18]. Thus, using the notation in [loc. cit.], we have $G=\mathbf{G}^{F}$ where $\mathbf{G}$ is a connected reductive algebraic group $\mathbf{G}$ over $\overline{\mathbb{F}}_{p}$ and $F: \mathbf{G} \rightarrow \mathbf{G}$ is an endomorphism such that some power of $F$ is a Frobenius map. Then the ingredients of the $B N$-pair in $G$ will also be derived from $\mathbf{G}$ : we have $B=\mathbf{B}^{F}$ where $\mathbf{B}$ is an $F$-stable Borel subgroup of $\mathbf{G}$ and $H=\mathbf{T}^{F}$ where $\mathbf{T}$ is an $F$-stable maximal torus contained in $\mathbf{B}$; furthermore, $N=N_{\mathbf{G}}(\mathbf{T})^{F}$ and $U=\mathbf{U}^{F}$ where $\mathbf{U}$ is the unipotent radical of $\mathbf{B}$. In this setting, one can single out a certain class of non-degenerate group homomorphisms $\sigma: U \rightarrow k^{\times}$, as follows.

The commutator subgroup $[\mathbf{U}, \mathbf{U}]$ is an $F$-stable closed connected normal subgroup of $\mathbf{U}$. We define the subgroup $U^{*}:=[\mathbf{U}, \mathbf{U}]^{F} \subseteq U$; then $[U, U] \subseteq U^{*}$. Furthermore, we shall fix a group homomorphism $\sigma: U \rightarrow k^{\times}$which is a regular character, that is, we have $U^{*} \subseteq \operatorname{ker}(\sigma)$ and the restriction of $\sigma$ to $U_{s}$ is non-trivial for all $s \in S$. (Such characters always exist; see [1, Section 8.1] and [2, Definition 14.27].) Then the corresponding module $\Gamma_{\sigma}=k G \underline{\mathfrak{u}}_{\sigma}$ is called a Gelfand-Graev module for $G$. Let $h \in H$ and $\sigma^{h}: U \rightarrow k^{\times}$be defined by $\sigma^{h}(u):=\sigma\left(h^{-1} u h\right)$ for $u \in U$. Then $\sigma^{h}$ also is a regular character and

$$
\underline{\mathfrak{u}}_{\sigma^{h}}=\sum_{u \in U} \sigma^{h}(u) u=h \underline{\mathfrak{u}}_{\sigma} h^{-1} \quad \text { for all } h \in H .
$$

Hence, right multiplication by $h^{-1}$ defines an isomorphism between the corresponding GelfandGraev modules $\Gamma_{\sigma}$ and $\Gamma_{\sigma^{h}}$.

Remark 4.1. Let $Z(G)$ be the center of $G$. Then $Z(G) \subseteq H$ and $Z(G)=Z(\mathbf{G})^{F}$; see $[1$, Proposition 3.6.8]. Assume now that $Z(\mathbf{G})$ is connected. Then there are precisely $|H / Z(G)|$ regular characters and they are all conjugate under the action of $H$; see [1, Proposition 8.1.2]. For any $h \in H$ we have

$$
\underline{\mathfrak{u}}_{\sigma} n_{0} \underline{\mathfrak{b}}=h \underline{\mathfrak{u}}_{\sigma} h^{-1} n_{0} \underline{\mathfrak{b}}=h \underline{\mathfrak{u}}_{\sigma} n_{0} h^{-1} \underline{\mathfrak{b}}=h \underline{\mathfrak{u}}_{\sigma} n_{0} \underline{\mathfrak{b}}
$$

and so $\mathscr{S}_{\sigma}=\mathscr{S}_{\sigma^{h}}$. It follows that $\mathscr{S}_{\sigma}=\mathscr{S}_{\sigma^{*}}=\mathscr{S}_{\sigma^{\prime}}$ for all regular characters $\sigma, \sigma^{\prime}$ of $U$, and we can denote this submodule simply by $\mathscr{S}_{0}$. By Proposition 3.1(iii), the simple module $D_{0}:=\mathscr{S}_{0} /\left(\mathscr{S} \cap \mathscr{S}_{0}^{\perp}\right)$ is now self-dual. Furthermore, we have

$$
\operatorname{dim} \mathscr{S}_{0} \geqslant|H / Z(G)|
$$

Indeed, we have $\underline{\mathfrak{u}}_{\sigma} n_{0} \underline{\mathfrak{b}} \in \mathscr{S}_{0}$ for all regular characters $\sigma$. Since pairwise distinct group homomorphisms $U \rightarrow k^{\times}$are linearly independent, the elements $\underline{\mathfrak{u}}_{\sigma} n_{0} \underline{\mathfrak{b}}$ (where $\sigma$ runs over all regular characters of $U$ ) form a set of $|H / Z(G)|$ linearly independent elements in $\mathscr{S}_{0}$. 
Example 4.2. Let $G=\mathrm{GL}_{n}(q)$ where $q$ is a prime power. Then our module $\mathscr{S}_{0}$ is $S_{\lambda}$ in James' notation [6, Definition 11.11], where $\lambda$ is the partition of $n$ with all parts equal to 1 . We claim that $\mathscr{S}_{0}=\mathrm{St}_{k}$ in this case.

Indeed, by [6, Theorem 16.5], $\operatorname{dim} \mathscr{S}_{0}$ is independent of the field $k$, as long as $\operatorname{char}(k) \neq p$. Since $\mathrm{St}_{\mathbb{Q}}$ is irreducible, we conclude that $\operatorname{dim} \mathscr{S}_{0}=\operatorname{dim} \mathrm{St}_{k}$ and the claim follows. Consequently, by Proposition 3.1(ii), $D_{0}$ is the unique simple quotient of $\mathrm{St}_{k}$. (The facts that $\mathscr{S}_{0}=\mathrm{St}_{k}$ and that this module has a unique simple quotient are also shown by Szechtman [9, Section 4].) However, $\operatorname{dim} D_{0}$ may certainly vary as the field $k$ varies; see the tables in [6, p. 107].

See $[3,4.14]$ for further examples where $\mathscr{S}_{0}=\mathrm{St}_{k}$. On the other hand, Gow [4, Section 5] gives examples (where $G=\mathrm{Sp}_{4}(q)$ ) where $\mathrm{St}_{k}$ does not have a unique simple quotient, and so $\mathscr{S}_{0} \varsubsetneqq \mathrm{St}_{k}$. Here is a further example.

Example 4.3. Let $G=\operatorname{Ree}\left(q^{2}\right)$ be the Ree group of type ${ }^{2} G_{2}$, where $q$ is an odd power of $\sqrt{3}$. Then $G$ has a $B N$-pair of rank 1 and so $[G: B]=\operatorname{dim} \mathrm{St}_{k}+1$. Let $k$ be a field of characteristic 2 . Then $k G \underline{\mathfrak{b}}$ and $\mathrm{St}_{k}$ have socle series as follows:

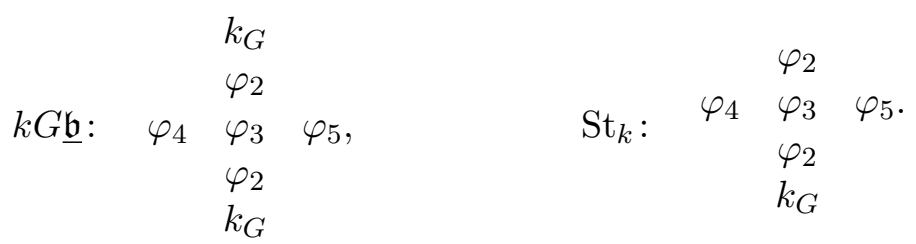

Here, $\varphi_{i}(i=1,2,3,4,5)$ are simple $k G$-modules and $\varphi_{4}$ is the contragredient dual of $\varphi_{5}$; see Landrock-Michler [7, Proposition 3.8(b)]. By Proposition 3.1, we have $D_{0} \cong \varphi_{3}$ and $\mathscr{S}_{0}$ is the uniserial submodule with composition factors $k_{G}, \varphi_{2}, \varphi_{3}$.

\section{References}

[1] Carter R.W., Finite groups of Lie type. Conjugacy classes and complex characters, Wiley Classics Library, John Wiley \& Sons, Ltd., Chichester, 1993.

[2] Digne F., Michel J., Representations of finite groups of Lie type, London Mathematical Society Student Texts, Vol. 21, Cambridge University Press, Cambridge, 1991.

[3] Geck M., On the modular composition factors of the Steinberg representation, J. Algebra 475 (2017), 370-391.

[4] Gow R., The Steinberg lattice of a finite Chevalley group and its modular reduction, J. London Math. Soc. 67 (2003), 593-608.

[5] Hiss G., Harish-Chandra series of Brauer characters in a finite group with a split BN-pair, J. London Math. Soc. 48 (1993), 219-228.

[6] James G.D., Representations of general linear groups, London Mathematical Society Lecture Note Series, Vol. 94, Cambridge University Press, Cambridge, 1984.

[7] Landrock P., Michler G.O., Principal 2-blocks of the simple groups of Ree type, Trans. Amer. Math. Soc. 260 (1980), 83-111.

[8] Steinberg R., Prime power representations of finite linear groups. II, Canad. J. Math. 9 (1957), $347-351$.

[9] Szechtman F., Steinberg lattice of the general linear group and its modular reduction, J. Group Theory 14 (2011), 603-635, arXiv:0812.2232.

[10] Tinberg N.B., The Steinberg component of a finite group with a split (B, N)-pair, J. Algebra 104 (1986), 126-134. 